\title{
Non-autonomous Hénon-Heiles Systems
}

\author{
Andrew N. W. Hone \\ Department of Mathematics and Statistics, \\ The University of Edinburgh, \\ Edinburgh, UK. \\ e-mail: hone@maths.ed.ac.uk
}

November 3, 2018

\begin{abstract}
Scaling similarity solutions of three integrable PDEs, namely the Sawada-Kotera, fifth order KdV and Kaup-Kupershmidt equations, are considered. It is shown that the resulting ODEs may be written as non-autonomous Hamiltonian equations, which are time-dependent generalizations of the well-known integrable Hénon-Heiles systems. The (time-dependent) Hamiltonians are given by logarithmic derivatives of the tau-functions (inherited from the original PDEs). The ODEs for the similarity solutions also have inherited Bäcklund transformations, which may be used to generate sequences of rational solutions as well as other special solutions related to the first Painlevé transcendent.
\end{abstract}




\section{Introduction}

The six Painlevé transcendents (PI-VI) have had a considerable amount of attention devoted to them in recent years, and have been studied from many different points of view. Their original discovery came about from Painlevé's classification of second order ODEs (of a particular form) having no movable critical points. They have also been approached by way of isomonodromic deformation of linear differential equations [14], or via abelian integrals and algebraic geometry [25]. Furthermore, they have found numerous physical applications. For example, the first Painlevé transcendent (PI),

$$
\frac{d^{2} w}{d z^{2}}=6 w^{2}+z
$$

arises as a string equation in the matrix models of 2-D gravity [12], while other Painlevé transcendents govern the behaviour of correlation functions in certain models of statistical mechanics [6], quantum field theory [22] and random matrices [18] (see chapter 7 of [3] for a more complete survey). Higher order ODEs of Painlevé type also occur in many situations, but as yet there is no complete classification of such equations.

Another context in which ODEs of Painlevé type arise naturally is as similarity reductions of integrable PDEs. A great deal of the renewed interest in Painlevé equations was generated by the conjecture of Ablowitz, Ramani and Segur [1], which states that all similarity reductions of integrable PDEs are of Painlevé type. For example, the modified Korteweg-deVries (mKdV) equation,

$$
v_{t}=v_{x x x}-6 v^{2} v_{x}
$$

has scaling similarity solutions of the form

$$
v(x, t)=(-3 t)^{-\frac{1}{3}} y(z(x, t)), \quad z=(-3 t)^{-\frac{1}{3}} x,
$$

where $y(z)$ satisfies

$$
y^{\prime \prime}=2 y^{3}+z y+\alpha,
$$

for some constant $\alpha$ (' denotes $\left.\frac{d}{d z}\right)$, which is the second Painlevé equation (PII). An important feature of the similarity reductions of soliton-type equations is that they inherit much of the stucture associated with integrability, such as Bäcklund transformations and solutions in terms of special functions.

In the work of Okamoto [27], each of the equations PI-VI is written as a non-autonomous Hamiltonian system, with a polynomial Hamiltonian, e.g. (1.3) may be obtained from the Hamiltonian

$$
h=\frac{1}{2} p^{2}-\left(y^{2}+\frac{z}{2}\right) p-\left(\alpha+\frac{1}{2}\right) y
$$


(where $y, p$ are the canonically conjugate variables, and $z$ denotes time). Each of the Hamiltonians used in [27 has explicit dependence on the time $z$, and is obtained from a holomorphic tau-function,

$$
h(z)=\frac{d}{d z} \log [\tau(z)]
$$

The tau-function provides a simple way to encode both the solutions of the equations and their Bäcklund transformations. In the case of PII, this taufunction essentially coincides with the tau-function of $\mathrm{mKdV} / \mathrm{KdV}$ (up to a factor of $\left.\exp \left[z^{3} / 24\right]\right)$. Manin [25] has found an alternative Hamiltonian description for PVI, involving the Weierstrass $\wp$ function, but his approach is difficult to generalize to higher order equations of Painlevé type.

In what follows we apply the approach of Okamoto, using polynomial Hamiltonians and tau-functions, in order to analyze the the scaling similarity solutions of three different integrable, fifth order evolution equations, namely the Sawada-Kotera (SK) equation,

$$
u_{t}=u_{5 x}+5 u u_{3 x}+5 u_{x} u_{x x}+5 u^{2} u_{x},
$$

fifth order KdV,

$$
u_{t}=u_{5 x}+10 u u_{3 x}+20 u_{x} u_{x x}+30 u^{2} u_{x}
$$

and the Kaup-Kupershmidt (KK) equation,

$$
u_{t}=u_{5 x}+10 u u_{3 x}+25 u_{x} u_{x x}+20 u^{2} u_{x}
$$

The ODEs for scaling similarity solutions of each of the flows in the SK, $\mathrm{KdV}$ and KK hierarchies, as well as their modified hierarchies (related by a Miura map), are presented in section 2. In the third section, by a direct extension of Fordy's results [16] on stationary flows, it is shown that the scaling similarity solutions of the fifth order equations in each hierarchy are related to non-autonomous generalizations of the integrable Hénon-Heiles systems. We describe the tau-functions, Miura maps and Bäcklund transformations associated to these systems, and use this machinery to generate sequences of special solutions in section 4 . The essence of our method is that the Hamiltonian formalism for stationary flows [17] may be extended to other (non-stationary) similarity reductions. We briefly discuss further developments in our concluding section.

Note. In their most basic form, these results were originally presented in [19], although these proceedings do not seem to be generally available 
at present; my thesis [20] contains a more thorough discussion. Shortly after my thesis was submitted, I was made aware of Kudryashov's paper [24] concerning similarity reductions in the $\mathrm{KdV} / \mathrm{mKdV}$ hierarchy. The results in [24 have some overlap with my own, but do not make use of the Hamiltonian formalism.

\section{Scaling Similarity Solutions in the Sawada- Kotera, KdV and Kaup-Kupershmidt Hi- erarchies}

In this section we develop a concise notation to describe the scaling similarity solutions of any one of the flows of the SK, KdV or KK hierarchies. We then apply it to some particular examples, including the similarity solutions of the fifth order equations which we study in detail in sections 3 and 4 .

\subsection{General Description of Scaling Similarity Solutions}

SK and KK have only one Hamiltonian structure, but KdV is bi-Hamiltonian, and here we will be using the second Hamiltonian structure. Following Fordy [16], we are able to consider all three hierarchies at once. The $n$th flow in each of the hierarchies can be written as

$$
\frac{\partial u}{\partial t_{n}}=\left(\partial_{x}^{3}+8 a u \partial_{x}+4 a u_{x}\right) \delta_{u} H_{n}[u],
$$

where $a=1 / 2$ for SK and $\mathrm{KdV}, a=1 / 4$ for $\mathrm{KK}$, and $H_{n}$ is the $n$th Hamiltonian density for the hierarchy in question. There is also a Miura map from the modified versions of the hierarchies, given by

$$
u=-v_{x}-2 a v^{2}=: M[v] .
$$

The $n$th flow of the modified hierarchy is

$$
\frac{\partial v}{\partial t_{n}}=\left(-\partial_{x}\right) \delta_{v} H_{n}[M[v]]
$$

The Miura map means that given $v$ satisfying (2.2) for each $n$, the corresponding $u=-v_{x}-2 a v^{2}$ satisfies (2.1). Further details on these hierarchies may be found in 15 .

The $n$th flow of the hierarchy is unchanged by the scaling

$$
x \rightarrow \beta x, \quad t_{n} \rightarrow \beta^{m} t_{n}, \quad u \rightarrow \beta^{-2} u,
$$


where $m=m(n)$ is a scale weight dependent on the hierarchy and on which flow is being considered. Similarly the modified flow is invariant under the same scaling but with

$$
v \rightarrow \beta^{-1} v .
$$

Hence there are scaling similarity solutions of the form

$$
u\left(x, t_{n}\right)=\theta^{2}\left(t_{n}\right) w(z), \quad z=x \theta\left(t_{n}\right), \quad \frac{d \theta}{d t_{n}}=\theta^{m+1} .
$$

(We use the notation of [11].) The corresponding similarity solution for the modified flow is

$$
v=\theta\left(t_{n}\right) y(z)
$$

with the scaled Miura map giving

$$
w=-y^{\prime}-2 a y^{2}=: \tilde{M}[y]
$$

(' denotes $\frac{d}{d z}$ throughout).

In the context of an integrable hierarchy, it is customary to think of the dependent variable as a function of all the times,

$$
u=u\left(t_{1}=x, t_{2}, t_{3}, \ldots\right) .
$$

For the scaling similarity solutions of the $n$th flow, it is necessary to drop dependence on anything other than $x$ and $t_{n}$, and it is inconsistent to consider the other flows simultaneously. To incorporate the other flows extra similarity variables must be included (e.g. in similarity reductions of the mKP equation [24). Such multi-phase similarity reductions are considered in chapter 4 of [26], and we will discuss how our approach extends to these solutions in [21]. Here we consider dependence on $x$ and $t_{n}$ only, and henceforth we drop the suffix $n$, bearing in mind that the actual form of $H$ depends on which particular flow we have chosen.

Substituting the similarity forms into the equations of motion (2.1) and (2.2) (and cancelling out powers of $\theta$ on either side) yields the ODEs for $w$ and $y$. If we let $\tilde{H}$ denote the rescaled $H$ (in terms of $w$ with powers of $\theta$ divided out) then we obtain the following:

$$
\begin{aligned}
\left(\partial^{3}+8 a w \partial+4 a w^{\prime}\right)\left(\delta_{w} \tilde{H}[w]-\frac{1}{4 a} z\right) & =0, \\
\partial\left(\delta_{y} \tilde{H}[\tilde{M}[y]]+z y\right) & =0 .
\end{aligned}
$$


The symbol $\partial$ denotes derivatives with respect to $z$. Both equations (2.3,2.4) can be integrated once, to yield

$$
\begin{aligned}
\frac{d^{2} f}{d z^{2}}+4 a w f+\frac{\lambda^{2}-\left(\frac{d f}{d z}\right)^{2}}{2 f} & =0, \\
\frac{d f}{d z}-4 a y f+\lambda & =0 .
\end{aligned}
$$

In the above, $\lambda$ is a constant of integration, and we have introduced the quantity

$$
f:=\delta_{w} \tilde{H}-\frac{1}{4 a} z .
$$

The equations (2.3, 2.4) deserve some comment, as we shall make use of them in section 3. With the definition (2.7), we may take $f=f[w]$, and thus (2.5) is to be considered as the ODE for $w$, obtained directly from (2.3) after an integration. However, using the scaled Miura map it is also consistent to take $f=f[\tilde{M}[y]]$, and so (by a slight abuse of notation) the equation (2.6) represents the ODE for the variable $y$, obtained by integrating (2.4). However, a more obvious direct integration of (2.4) would be

$$
\delta_{y} \tilde{H}+z y+\alpha=0,
$$

for some constant $\alpha$. To show that (2.6) is equivalent to (2.8), it is necessary to use the scaled version of the identity

$$
\delta_{v} H=\left(M^{\prime}\right)^{*} \delta_{u} H=\left(\partial_{x}-4 a v\right) \delta_{u} H
$$

(with $M^{\prime}$ being the Fréchet derivative of $M$ ), and then it is found that the constants of integration are related by

$$
\alpha=\lambda-\frac{1}{4 a}
$$

Another important point is that the same constant $\lambda$ appears in both (2.5) and (2.6). This is because the Miura map for the original PDEs (2.1,2.2) becomes a one-one correspondence between the ODEs for their scaling similarity solutions. The scaled Miura map is

$$
w=-y^{\prime}-2 a y^{2},
$$

and it has an inverse given by

$$
y=\frac{f^{\prime}+\lambda}{4 a f} .
$$


In (2.10) $f$ should be regarded as $f[w]$, and it is necessary to assume $f \not \equiv 0$ since otherwise this equation breaks down. It is straightforward to see the one-one correspondence between (2.5) and (2.6). Indeed, given a solution $w$ of (2.5), the modified variable $y$ may be defined by the inverse Miura map (2.10). A direct calculation then shows that the Miura formula (2.9) holds, meaning that $f$ can be reinterpreted as $f[\tilde{M}[y]]$. Thus (on rearranging $(2.10)$ )

$y$ satisfies the ODE (2.6); the converse follows by reversing this argument (or by scaling the Miura map for the PDEs). To make things more concrete, it is worth looking at some particular cases.

\subsection{Similarity Solutions of KdV and PII}

The correspondence between PII (the equation (1.3)) and scaling similarity solutions of the ordinary $\mathrm{KdV}$ equation is well-known. Taking $H=\frac{1}{2} u^{2}$ in (2.1) with $a=1 / 2$ yields $\mathrm{KdV}$,

$$
u_{t}=u_{x x x}+6 u u_{x}
$$

with scaling similarity solutions

$$
u(x, t)=(-3 t)^{-\frac{2}{3}} w(z), z=(-3 t)^{-\frac{1}{3}} x .
$$

The scaled Hamiltonian $\tilde{H}=\frac{1}{2} w^{2}$ gives

$$
f[w]=w-\frac{z}{2}
$$

whence (2.5) with $a=\frac{1}{2}$ becomes

$$
w^{\prime \prime}+2 w^{2}-z w+\frac{\alpha(\alpha+1)+w^{\prime}-\left(w^{\prime}\right)^{2}}{2 w-z}=0,
$$

taking $\lambda=\alpha+\frac{1}{2}$.

For the scaling similarity solutions of $\mathrm{mKdV}(1.2)$, the one-one correspondence between solutions of PII and (2.12) is given by

$$
w=-y^{\prime}-y^{2}
$$

(the scaled Miura map) and

$$
y=\frac{w^{\prime}+\alpha}{2 w-z}
$$

the latter being a particular case of the inverse Miura formula (2.10). Observe that, in terms of $y$, we have

$$
f[\tilde{M}[y]]=-y^{\prime}-y^{2}-\frac{z}{2},
$$

and on putting this into (2.6) with $a=\frac{1}{2}$, PII results. This example is also considered in [2], for instance. 


\subsection{Similarity Solutions of Fifth Order Equations}

Our second example constitutes the main subject of sections 3 and 4. Following [16], we use the equation (2.1) with Hamiltonian density

$$
H=-\frac{1}{2} u_{x}^{2}-\frac{1}{3} b u^{3}
$$

leading to the fifth order equation

$$
u_{t}=\left(u_{x x x x}+(8 a-2 b) u u_{x x}-2(a+b) u_{x}^{2}-\frac{20}{3} a b u^{3}\right)_{x} .
$$

There are only three cases (i),(ii),(iii), corresponding to

$$
a=\frac{1}{2}, \quad \frac{1}{2}, \quad \frac{1}{4}, \quad b=-\frac{1}{2}, \quad-3, \quad-4,
$$

for which an equation of the form (2.13) is integrable, and these three cases correspond to the SK equation (1.5), the fifth order KdV equation (1.6), and the KK equation (1.7) respectively. When specializing to one of the three hierarchies, it is necessary to take the correct values of $a$ and $b$ in each case. While some of the calculations we present are valid for arbitrary $a, b$, all properties relevant to the integrability of the equations are lost in general.

The scaling similarity solutions of (2.13) take the form

$$
u(x, t)=(-5 t)^{-\frac{2}{5}} w(z), z=(-5 t)^{-\frac{1}{5}} x .
$$

From the scaled density $\tilde{H}$ we find

$$
f[w]=w^{\prime \prime}-b w^{2}-\frac{z}{4 a},
$$

and substituting this into (2.5) yields a fourth order ODE for $w$, which we prefer not to present explicitly. In the next section we show that this ODE for $w$ is equivalent to a non-autonomous Hamiltonian system of Hénon-Heiles type.

Scaling similarity solutions of the associated modified equations take the form

$$
v(x, t)=(-5 t)^{-\frac{1}{5}} y(z) .
$$

Using the scaled Miura map, we may express $f$ in terms of the modified variable $y$ :

$$
f[\tilde{M}[y]]=-y^{\prime \prime \prime}-4 a y y^{\prime \prime}-(4 a+b)\left(y^{\prime}\right)^{2}-4 a b y^{2} y^{\prime}-4 a^{2} b y^{4}-\frac{z}{4 a} .
$$


Then the ODE for $y$ is (from (2.6))

$$
y^{(i v)}=-2(6 a+b) y^{\prime} y^{\prime \prime}+4 a(4 a-b)\left(y^{2} y^{\prime \prime}+y\left(y^{\prime}\right)^{2}\right)+16 a^{3} b y^{5}+z y+\alpha,
$$

with

$$
\alpha=\lambda-\frac{1}{4 a} .
$$

Given a solution to (2.16) we can then obtain a solution to the fourth order ODE for $w$, via $w=-y^{\prime}-2 a y^{2}$ (and vice-versa, using the inverse Miura map (2.10)).

On substituting in the relevant values of $a$ and $b$ for the integrable cases (i),(ii),(iii) into the equation (2.16) we find

$$
\begin{aligned}
& y^{(i v)}=-5 y^{\prime} y^{\prime \prime}+5\left(y^{2} y^{\prime \prime}+y\left(y^{\prime}\right)^{2}\right)-y^{5}+z y+\alpha, \\
& y^{(i v)}=10\left(y^{2} y^{\prime \prime}+y\left(y^{\prime}\right)^{2}\right)-6 y^{5}+z y+\alpha, \\
& y^{(i v)}=5 y^{\prime} y^{\prime \prime}+5\left(y^{2} y^{\prime \prime}+y\left(y^{\prime}\right)^{2}\right)-y^{5}+z y+\alpha
\end{aligned}
$$

the ODEs for scaling similarity solutions of the modified SK, fifth order $\mathrm{mKdV}$ and modified KK equations respectively. Notice that (2.17) and (2.19) differ only by a sign in the even $\left(y^{\prime} y^{\prime \prime}\right)$ terms. Hence if $y_{(i)}$ is as solution to (2.16) for case (i), then $y_{(i i i)}=-y_{(i)}$ will be a solution to that equation for case (iii) with $\alpha$ replaced by $-\alpha$. This is because the modified hierarchies in these two cases are essentially the same; both SK and KK have a third order Lax operator, which factorizes to yield the Miura map (see [15]). In the next section we shall use this connection between case (i) and case (iii) to derive the Bäcklund transformations for these scaling similarity equations, after relating them to Hénon-Heiles systems.

Remark. In 24] the sequence of equations (2.6) are referred to as the second Painlevé equations of $2 n$th order (corresponding to scaling similarity solutions to the flow given by $H_{n+1}$ ). For instance, (up to a rescaling) the equation (2.18) is the second Painlevé equation of fourth order in the nomenclature of [24]. However, this sequence of ODEs is by no means new; it appears in [14 under the name of the Painlevé II Family.

\section{Hénon-Heiles Systems}

\subsection{Stationary Flows and Integrable Hénon-Heiles}

The original Hénon-Heiles system is given by a Hamiltonian with two degrees

of freedom: 


$$
h=\frac{1}{2}\left(p_{1}^{2}+p_{2}^{2}\right)+a q_{1} q_{2}^{2}-\frac{1}{3} b q_{1}^{3},
$$

The equations of motion are just Hamilton's equations,

$$
\frac{d q_{j}}{d z}=\frac{\partial h}{\partial p_{j}}, \quad \frac{d p_{j}}{d z}=-\frac{\partial h}{\partial q_{j}} .
$$

(We are denoting the time by $z$ here to make connection with our other results.) It has been known for some time from Painlevé analysis 10 that this system is integrable for only three values of the ratio $r=a / b$, namely

$$
r=-1, \quad-1 / 6, \quad-1 / 16
$$

More recently it was shown by Fordy [16] that in these integrable cases the equations of motion are just disguised versions of the stationary flows of the SK, fifth order KdV and KK equations. Thus the special values of $a$ and $b$ listed in the previous section give the right values for the ratio $r$ in the cases (i),(ii),(iii) respectively. The zero curvature form of these PDEs yields a matrix Lax representation of the stationary flows, and then traces of powers of the Lax matrix give the Hamiltonian and a second constant of motion in involution (implying Liouville integrability). It was subsequently shown that all three Hamiltonian systems are completely separable in suitable coordinates, and may be integrated in terms of theta functions of genus one (cases (i) \& (iii)) or genus two (case (ii)) [9].

\subsection{Non-autonomous Hamiltonians for Scaling Simi- larity Solutions}

In order to consider the ODEs for scaling similarity solutions of the three integrable fifth order PDEs (all of the form (2.13)), we adapt Fordy's approach [16] to the stationary flows, and write them in Hamiltonian form. Recall that the previous section we have the quantity $f$ given by

$$
f=w^{\prime \prime}-b w^{2}-\frac{z}{4 a}
$$

and on substituting this into (2.5) a full fourth order ODE for $w$ results. Instead of presenting this explicitly, we set

$$
w=q_{1}, \quad f=-a q_{2}^{2}
$$


the latter is a squared eigenfunction substitution [8]. Then we may rewrite (2.5,3.3) as a coupled system for $q_{1}, q_{2}$ :

$$
\begin{aligned}
& q_{1}^{\prime \prime}=b q_{1}^{2}-a q_{2}^{2}+\frac{z}{4 a}, \\
& q_{2}^{\prime \prime}=-2 a q_{1} q_{2}-\frac{\lambda^{2}}{4 a^{2} q_{2}^{3}} .
\end{aligned}
$$

This coupled system just follows from Hamilton's equations (3.2), where now the Hamiltonian is given by

$$
h=\frac{1}{2}\left(p_{1}^{2}+p_{2}^{2}\right)+a q_{1} q_{2}^{2}-\frac{1}{3} b q_{1}^{3}-\frac{\lambda^{2}}{8 a^{2}} q_{2}^{-2}-\frac{1}{4 a} z q_{1} .
$$

Compared with (3.1), this has an extra inverse square term and a nonautonomous (time-dependent) term in the potential.

Thus we have shown that the equations for the similarity solutions of (2.13) may be viewed as non-autonomous Hénon-Heiles systems. Because of the explicit time-dependence, the Hamiltonian $h(z)$ satisfies a fourth order ODE which (in the generic situation $f^{\prime} \not \equiv 0$ ) is equivalent to the system (3.4,3.5) (this is proved in [20]). Applying Painlevé analysis to this system (which is almost identical to the analysis for the autonomous case [10]), it is found that the Painlevé test is only satisfied for the three special values of the ratio $r$ (which correspond to the scaling similarity solutions of the integrable PDEs). Because they are similarity reductions of PDEs solvable by inverse scattering, these special cases can also be derived from a zero curvature condition [20], and solved by the inverse monodromy method [14], but we will not consider this approach here.

For the particular special choices of $a$ and $b$ given in (2.14), we denote the Hamiltonian systems (with Hamiltonian (3.6)) by $\mathcal{H}_{(i)}, \mathcal{H}_{(i i)}, \mathcal{H}_{(i i i)}$ (corresponding to the reductions of SK, KdV and KK respectively). Each system has Bäcklund transformations which can be viewed as a canonical transformations in the variables $q_{j}, p_{j}, z$, i.e. under the transformation

$$
\left(q_{j}, p_{j}, z\right) \longrightarrow\left(\tilde{q}_{j}, \tilde{p}_{j}, \tilde{z}\right)
$$

the two-form

$$
\omega=\sum_{j=1,2} d p_{j} \wedge d q_{j}-d h \wedge d z
$$

is preserved.

The construction of the Bäcklund transformations is possible due to the existence of the Miura maps. For the system $\mathcal{H}_{(i i)}$ we have (by the general arguments of section 2) a one-one correspondence between its solutions and 
the solutions of the modified similarity equation (2.18), while both $\mathcal{H}_{(i)}$ and $\mathcal{H}_{(i i i)}$ have similar correspondences with (2.17). We are also able to define a tau-function for the Hamiltonians, via

$$
h\left(q_{j}(z), p_{j}(z), z\right) \propto(\log [\tau(z)])^{\prime}
$$

(for some constant of proportionality, dependent on which of the three cases is being considered). We consider the system $\mathcal{H}_{(i i)}$ first, as this is the simplest, while the systems $\mathcal{H}_{(i),(i i i)}$ are then considered in tandem (because they are connected via the correspondence with the same modified equation (2.17)).

\subsection{The Hamiltonian System $\mathcal{H}_{(i i)}$}

The Hamiltonian for $\mathcal{H}_{(i i)}$ is given explicitly by

$$
h_{\lambda}=\frac{1}{2}\left(p_{1}^{2}+p_{2}^{2}\right)+\frac{1}{2} q_{1} q_{2}^{2}+q_{1}^{3}-\frac{\lambda^{2}}{2} q_{2}^{-2}-\frac{1}{2} z q_{1}
$$

We will henceforth put suffixes on all quantities to denote their dependence on $\lambda$, as the Bäcklund transformation relates the same quantities for different values of this parameter. There is also the alternative parameter $\alpha=\lambda-\frac{1}{2}$ appearing in (2.18), but $\lambda$ is the more natural one in that (3.8) depends only on $\lambda^{2}$, i.e.

$$
h_{\lambda}=h_{-\lambda},
$$

and thus the same is true for the solutions $\left(q_{j}(z), p_{j}(z)\right)$ to the system with Hamiltonian $h_{\lambda}$. The most convenient variables to use for this system are

$$
w_{\lambda}=q_{1}, \quad f_{\lambda}=-\frac{1}{2} q_{2}^{2},
$$

where (by (3.3) with $a=1 / 2, b=-3$ ) we have

$$
f_{\lambda}=w_{\lambda}^{\prime \prime}+3 w_{\lambda}^{2}-\frac{z}{2}
$$

Using the Miura map, there is a one-one correspondence with solutions to (2.18). In other words, given a solution to the Hamiltonian system, we find a solution $y=y_{\lambda}$ to equation (2.18) (with $\alpha=\lambda-\frac{1}{2}$ ) via the formula (2.10),

$$
y_{\lambda}=\frac{f_{\lambda}^{\prime}+\lambda}{2 f_{\lambda}} .
$$

We also have the usual Miura expression,

$$
w_{\lambda}=-y_{\lambda}^{\prime}-y_{\lambda}^{2},
$$


which means that conversely a solution to (2.18) determines a solution to the system $\mathcal{H}_{(i i)}$. Before presenting the Bäcklund transformation, it is helpful to introduce the tau-function.

Definition 1 For a solution $\left(q_{j}(z), p_{j}(z)\right)$ of the system $\mathcal{H}_{(i i)}$, we have the Hamiltonian $h_{\lambda}(z)=h_{\lambda}\left(q_{j}(z), p_{j}(z), z\right)$. The tau-function associated with this solution is given by

$$
h_{\lambda}(z)=-\frac{d}{d z} \log \left[\tau_{\lambda}(z)\right]
$$

The above definition is chosen to be consistent with the tau-function of the $\mathrm{KdV}$ hierarchy, where the dependent variable $u$ is expressed as

$$
u(x, t)=2(\log [\tau(x, t)])_{x x} .
$$

For scaling similarity solutions we require that $\tau$ depends on $x$ and $t$ only through the combination $z=x \theta(t)$, which means we must have

$$
w_{\lambda}=2(\log [\tau(z)])^{\prime \prime} .
$$

This agrees with our definition, for differentiating the Hamiltonian gives

$$
-(\log [\tau(z)])^{\prime \prime}=h_{\lambda}^{\prime}=-\frac{1}{2} q_{1}=-\frac{1}{2} w_{\lambda}
$$

Proposition 1 The Bäcklund transformation for the equation (2.18) may be written in the form

$$
y_{\lambda+1}=-y_{\lambda}+\frac{\lambda}{f_{\lambda}}
$$

Moreover, this induces a canonical transformation from the system $\mathcal{H}_{(i i)}$ with parameter $\lambda$ to the same system with parameter $\lambda+1$ :

$$
q_{j} \rightarrow \tilde{q}_{j}, \quad p_{j} \rightarrow \tilde{p}_{j}, \quad z \rightarrow z, \quad h_{\lambda} \rightarrow h_{\lambda+1} .
$$

The modified variable $y_{\lambda}$ may also be written in terms of the two tau-functions related by this transformation:

$$
y_{\lambda}=\left(\log \left[\tau_{\lambda-1} / \tau_{\lambda}\right]\right)^{\prime}
$$

Proof. The first thing to observe is that $w_{\lambda}$ is related to two different modified variables by the Miura map (3.11):

$$
w_{\lambda}=-y_{\lambda}^{\prime}-y_{\lambda}^{2}=-y_{-\lambda}^{\prime}-y_{-\lambda}^{2} .
$$


This is a well-known property of Miura maps, but can be seen directly from the fact that the fourth order ODE for $w_{\lambda}$ just depends on $\lambda^{2}$, so

$$
w_{\lambda}=w_{-\lambda}
$$

The inverse Miura map (3.10) gives

$$
y_{ \pm \lambda}=\frac{f_{\lambda}^{\prime} \pm \lambda}{2 f_{\lambda}}
$$

the solutions to (2.18) for parameter $\alpha= \pm \lambda-\frac{1}{2}$. Looking at the modified equation (2.18), we see that it is unchanged on sending

$$
y \rightarrow-y, \quad \alpha \rightarrow-\alpha .
$$

Hence $-y_{-\lambda}$ will be a solution to this equation for $\alpha=\lambda+\frac{1}{2}=(\lambda+1)-\frac{1}{2}$, or in other words we may identify

$$
y_{\lambda+1}=-y_{-\lambda} .
$$

It is then straightforward to derive (3.13) using the inverse Miura formula. This same argument also works for PII and the rest of the PII Family. Note that $f_{\lambda}$ in the right hand side of the Bäcklund transformation may be written in terms of $y_{\lambda}$ and its derivatives.

To define the induced transformation of the Hamiltonian system $\mathcal{H}_{(i i)}$, first of all it is necessary to write $y_{\lambda+1}$ in terms of the variables appearing in $h_{\lambda}$ :

$$
y_{\lambda+1}=-\frac{p_{2} q_{2}+\lambda}{q_{2}^{2}} .
$$

The Miura map produces a new solution to the fourth order equation (2.5) at parameter value $\lambda+1$,

$$
w_{\lambda+1}=-y_{\lambda+1}^{\prime}-y_{\lambda+1}^{2} .
$$

Now we can define the new variables $\left(\tilde{q}_{j}, \tilde{p}_{j}\right)$ via

$$
\tilde{q}_{1}=w_{\lambda+1}, \quad \tilde{p}_{1}=w_{\lambda+1}^{\prime}, \quad \tilde{q}_{2}=\left(-2 f_{\lambda+1}\right)^{\frac{1}{2}}, \quad \tilde{p}_{2}=-\left(-2 f_{\lambda+1}\right)^{-\frac{1}{2}} f_{\lambda+1}^{\prime},
$$

and these will clearly satisfy the system $\mathcal{H}_{(i i)}$ with Hamiltonian $h_{\lambda+1}$. It is simple to write the new variables in terms of the old (and vice-versa), and we present the formulae here for completeness:

$$
\begin{array}{ll}
\tilde{q}_{1}=-q_{1}-2 y_{\lambda+1}^{2}, & q_{1}=-\tilde{q}_{1}-2 y_{\lambda+1}^{2}, \\
\tilde{p}_{1}=-p_{1}-4 y_{\lambda+1}\left(q_{1}+y_{\lambda+1}^{2}\right), & p_{1}=-\tilde{p}_{1}+4 y_{\lambda+1}\left(\tilde{q}_{1}+y_{\lambda+1}^{2}\right), \\
\tilde{q}_{2}=\Upsilon^{\frac{1}{2}}, & q_{2}=\tilde{\Upsilon}^{\frac{1}{2}}, \\
\tilde{p}_{2}=y_{\lambda+1} \Upsilon^{\frac{1}{2}}+(\lambda+1) \Upsilon^{-\frac{1}{2}}, & p_{2}=-y_{\lambda+1} \tilde{\Upsilon}^{\frac{1}{2}}-\lambda \tilde{\Upsilon}^{-\frac{1}{2}},
\end{array}
$$


where

$\Upsilon=-q_{2}^{2}+8 y_{\lambda+1} p_{1}+8 y_{\lambda+1}^{2} q_{1}-4 q_{1}^{2}+2 z, \tilde{\Upsilon}=-\tilde{q}_{2}^{2}-8 y_{\lambda+1} \tilde{p}_{1}+8 y_{\lambda+1}^{2} \tilde{q}_{1}-4 \tilde{q}_{1}^{2}+2 z$,

and in the left-hand formulae $y_{\lambda+1}$ is to be interpreted as the function of $p_{2}$ and $q_{2}$ given by (3.16), while on the right-hand side take

$$
y_{\lambda+1}=\frac{\tilde{p}_{2} \tilde{q}_{2}-(\lambda+1)}{\tilde{q}_{2}^{2}} .
$$

For the sake of clarity, we present the (invertible) transformations in the variables $w_{\lambda}$ and $y_{\lambda}$ diagramatically:

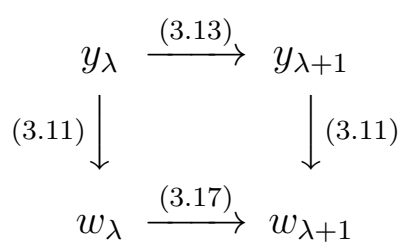

It is obvious that (3.17) is a canonical transformation, since the equations for both sets of variables are Hamiltonian; it is also possible to verify explicitly that the two-form (3.7) is preserved. A direct calculation also shows that difference in the Hamiltonians is

$$
h_{\lambda+1}-h_{\lambda}=y_{\lambda+1},
$$

which yields (3.14) immediately. This completes the proof.

\subsection{The Hamiltonian Systems $\mathcal{H}_{(i)}$ and $\mathcal{H}_{(i i i)}$}

The Hamiltonian for $\mathcal{H}_{(i)}$ is

$$
h_{\lambda}=\frac{1}{2}\left(p_{1}^{2}+p_{2}^{2}\right)+\frac{1}{2} q_{1} q_{2}^{2}+\frac{1}{6} q_{1}^{3}-\frac{\lambda^{2}}{2} q_{2}^{-2}-\frac{1}{2} z q_{1},
$$

while for $\mathcal{H}_{(i i i)}$ it is

$$
H_{\lambda}=\frac{1}{2}\left(P_{1}^{2}+P_{2}^{2}\right)+\frac{1}{4} Q_{1} Q_{2}^{2}+\frac{4}{3} Q_{1}^{3}-2 \lambda^{2} Q_{2}^{-2}-z Q_{1} .
$$

To avoid confusion between the two, we use lower/upper case letters for the variables of the systems $\mathcal{H}_{(i)} / \mathcal{H}_{(i i i)}$ respectively. It is convenient to use the variables

$$
w_{\lambda}=q_{1}, \quad f_{\lambda}=-\frac{1}{2} q_{2}^{2}, \quad W_{\lambda}=Q_{1}, \quad F_{\lambda}=-\frac{1}{4} Q_{2}^{2} .
$$


Hence, using (3.3) with the appropriate values of $a$ and $b$,

$$
f_{\lambda}=w_{\lambda}^{\prime \prime}+\frac{1}{2} w_{\lambda}^{2}-\frac{z}{2}, \quad F_{\lambda}=W_{\lambda}^{\prime \prime}+4 W_{\lambda}^{2}-z
$$

For case (i) the Miura map and its inverse are given by

$$
w_{\lambda}=-y_{\lambda}^{\prime}-y_{\lambda}^{2}, \quad y_{\lambda}=\frac{f_{\lambda}^{\prime}+\lambda}{2 f_{\lambda}}
$$

where $y_{\lambda}$ is a solution to (2.17) for $\alpha=\lambda-\frac{1}{2}$. Although case (iii) is related to the same modified equation, it will be helpful for deriving the Bäcklund transformation to return to the original formalism of Section 2, where the case (iii) (with $a=1 / 4$ ) the Miura map and its inverse are given by

$$
W_{\lambda}=-Y_{\lambda}^{\prime}-\frac{1}{2} Y_{\lambda}^{2}, \quad Y_{\lambda}=\frac{F_{\lambda}^{\prime}+\lambda}{F_{\lambda}}
$$

where $Y_{\lambda}$ is a solution to (2.19) for $\alpha=\lambda-1$. The derivation of the Bäcklund transformation for the equation (2.17) (or equivalently (2.19)) is most easily achieved with the tau-functions, naturally related to the tau-functions of the SK/KK hierarchies (see 28] for definitions of these).

Definition 2 For a solution $\left(q_{j}(z), p_{j}(z)\right)$ of the system $\mathcal{H}_{(i)}$, with the Hamiltonian $h_{\lambda}(z)=h_{\lambda}\left(q_{j}(z), p_{j}(z), z\right)$, the associated tau-function is given by

$$
h_{\lambda}(z)=-3 \frac{d}{d z} \log \left[\tau_{\lambda}(z)\right]
$$

Definition 3 For a solution $\left(Q_{j}(z), P_{j}(z)\right)$ of the system $\mathcal{H}_{(i i i)}$, with the Hamiltonian $H_{\lambda}(z)=H_{\lambda}\left(Q_{j}(z), P_{j}(z), z\right)$, the associated tau-function is given by

$$
H_{\lambda}(z)=-\frac{3}{2} \frac{d}{d z} \log \left[\tilde{\tau}_{\lambda}(z)\right]
$$

Now we may show:

Proposition 2 The Bäcklund transformation for the equation (2.17) may be written in the form

$$
y_{\lambda+3}=y_{\lambda}-\frac{\lambda}{f_{\lambda}}+\frac{2\left(\lambda+\frac{3}{2}\right)}{F_{\lambda+\frac{3}{2}}} .
$$


This is related to a canonical transformation between the system $\mathcal{H}_{(i)}$ with parameter $\lambda$ and the system $\mathcal{H}_{(i i i)}$ with parameter $\lambda-\frac{3}{2}$ :

$$
q_{j} \rightarrow Q_{j}, \quad p_{j} \rightarrow P_{j}, \quad z \rightarrow z, \quad h_{\lambda} \rightarrow H_{\lambda-\frac{3}{2}} .
$$

The modified variable $y_{\lambda}$ may also be written in terms of the two tau-functions related by this transformation:

$$
y_{\lambda}=\left(\log \left[\tilde{\tau}_{\lambda-\frac{3}{2}} / \tau_{\lambda}^{2}\right]\right)^{\prime} .
$$

Proof. As for case (ii), $w_{\lambda}$ (and also $W_{\lambda}$ ) may be related to two different modified variables by the Miura map. Hence we see that

$$
\begin{gathered}
y_{\lambda}-y_{-\lambda}=\frac{\lambda}{f_{\lambda}}, \\
Y_{\lambda}-Y_{-\lambda}=\frac{2 \lambda}{F_{\lambda}} .
\end{gathered}
$$

Clearly (3.25) constitutes a Bäcklund transformation for (2.17), as it relates two solutions for different parameter values. However, it is not very useful because it cannot be iterated to obtain a sequence of solutions. To achieve this requires a canonical tranformation from $\mathcal{H}_{(i)}$ to $\mathcal{H}_{(i i i)}$, and then from $\mathcal{H}_{(i i i)}$ back to $\mathcal{H}_{(i)}$ with the overall change $\lambda \rightarrow \lambda+3$. First of all observe that on comparing the parameters $\alpha$ in (2.17) and (2.19), it is apparent that we may identify

$$
y_{\lambda}=-Y_{-\lambda+\frac{3}{2}},
$$

and so the Miura map for case (iii) implies

$$
W_{-\lambda+\frac{3}{2}}=y_{\lambda}^{\prime}-\frac{1}{2} y_{\lambda}^{2}
$$

Since $W_{-\lambda+\frac{3}{2}}$ may be found from $w_{\lambda}$, via the formula

$$
W_{-\lambda+\frac{3}{2}}=-w_{\lambda}-\frac{3}{2} y_{\lambda}^{2}
$$

it is obvious that there is an induced canonical transformation from the system with Hamiltonian $h_{\lambda}$ to the system with Hamiltonian $H_{-\lambda+\frac{3}{2}}=H_{\lambda-\frac{3}{2}}$. This transformation and its inverse may be calculated explicitly in terms of coordinates, i.e.

$$
\begin{array}{ll}
Q_{1}=-q_{1}-\frac{3}{2} y_{\lambda}^{2}, & q_{1}=-Q_{1}-\frac{3}{2} y_{\lambda}^{2}, \\
P_{1}=-p_{1}+3 y_{\lambda}\left(q_{1}+y_{\lambda}^{2}\right), & p_{1}=-P_{1}-3 y_{\lambda}\left(Q_{1}+\frac{1}{2} y_{\lambda}^{2}\right), \\
Q_{2}=\aleph^{\frac{1}{2}}, & q_{2}=\tilde{\aleph}^{\frac{1}{2}} \\
P_{2}=-\frac{1}{2} y_{\lambda} \aleph^{\frac{1}{2}}-2\left(\lambda-\frac{3}{2}\right) \aleph^{-\frac{1}{2}}, & p_{2}=y_{\lambda} \tilde{\aleph}^{\frac{1}{2}}+\lambda \tilde{\aleph}^{-\frac{1}{2}},
\end{array}
$$


where

$\aleph=-2 q_{2}^{2}-12 y_{\lambda} p_{1}-6 q_{1}^{2}+6 z, \tilde{\aleph}=-\frac{1}{2} Q_{2}^{2}+6 y_{\lambda} P_{1}-3 Q_{1}^{2}+9 y_{\lambda}^{2} Q_{1}+\frac{9}{4} y_{\lambda}^{4}+3 z$,

and in the above $y_{\lambda}$ is to be interpreted alternately as a function of $p_{2}, q_{2}$ and $P_{2}, Q_{2}$ :

$$
y_{\lambda}=\frac{p_{2} q_{2}-\lambda}{q_{2}^{2}}=-\frac{2 P_{2} Q_{2}+4\left(\lambda-\frac{3}{2}\right)}{Q_{2}^{2}} .
$$

This is the analogue of the transformation between autonomous Hénon-Heiles systems considered in [8, 13].

The tau-function formula (3.24) follows directly from a calculation of the difference of the two Hamiltonians:

$$
h_{\lambda}-H_{\lambda-\frac{3}{2}}=\frac{3}{2} y_{\lambda}
$$

Then in terms of tau-functions, we have

$$
y_{\lambda+3}-y_{\lambda}=\frac{d}{d z}\left(\log \left[\tilde{\tau}_{\lambda+\frac{3}{2}} / \tilde{\tau}_{\lambda-\frac{3}{2}}\right]+2 \log \left[\tau_{\lambda} / \tau_{\lambda+3}\right]\right) .
$$

On using the formulae (3.25, 3.26), the Bäcklund transformation (3.23) follows. Thus overall there is an induced canonical transformation from $\mathcal{H}_{(i)}$ to itself, with the parameter $\lambda \rightarrow \lambda+3$, as can be seen from a diagram:

$$
\begin{aligned}
& y_{\lambda} \stackrel{\text { (3.25) }}{\longrightarrow} y_{-\lambda}=-Y_{\lambda+\frac{3}{2}} \stackrel{(3.26)}{\longrightarrow} Y_{-\lambda-\frac{3}{2}}=-y_{\lambda+3} \\
& \text { (3.21) } \downarrow \quad 3.21 \downarrow \quad 3.22 \downarrow \quad \text { (3.22) } \downarrow \quad \text { (3.21) } \downarrow \\
& w_{\lambda}=w_{-\lambda} \underset{\sqrt{3.27})}{\longrightarrow} W_{\lambda+\frac{3}{2}}=W_{-\lambda-\frac{3}{2}} \underset{(3.27)}{\longrightarrow} w_{\lambda+3}
\end{aligned}
$$

Note that in the right hand side of (3.23), $f_{\lambda}$ may be determined entirely in terms of $y_{\lambda}$ and its derivatives, and similarly for $F_{\lambda+\frac{3}{2}}$.

\subsection{Analogues of the Toda Lattice for Sequences of Tau-Functions}

It is well known [23, 27 that for PII, the tau-functions related by the Bäcklund transformation (after rescaling) satisfy the Toda lattice equation,

$$
D_{z}^{2} \tau_{\lambda} \cdot \tau_{\lambda}=\tau_{\lambda-1} \tau_{\lambda+1}
$$

The tau-functions of (2.18) (related by the Bäcklund transformation) also satisfy a bilinear lattice equation, which is a fourth order analogue of (3.28). 
Indeed, on the one hand (substituting for $w_{\lambda}$ in terms of $\tau_{\lambda}$ into (3.9)) we have

$$
f_{\lambda}=\tau_{\lambda}^{-2}\left(D_{z}^{4} \tau_{\lambda} \cdot \tau_{\lambda}\right)-\frac{z}{2}
$$

while on the other hand (from (3.14) and (3.15))

$$
\left(\log \left[\tau_{\lambda+1} \tau_{\lambda-1} / \tau_{\lambda}^{2}\right]\right)^{\prime}=y_{\lambda}+y_{-\lambda}=\left(\log \left[f_{\lambda}\right]\right)^{\prime}
$$

Thus integrating left- and right-hand sides of (3.30) and comparing with (3.29) yields the bilinear equation

$$
2 D_{z}^{4} \tau_{\lambda} \cdot \tau_{\lambda}-z \tau_{\lambda}^{2}=k_{\lambda} \tau_{\lambda-1} \tau_{\lambda+1}
$$

Note that the constant of integration $k_{\lambda}$ may be rescaled arbitrarily, since the tau-functions can always be rescaled (without affecting $h_{\lambda}, y_{\lambda}$ ). We take the convention $k_{\lambda}=1$, which ensures that the tau-functions for the rational solutions (presented in section 4) are monic polynomials in $z$. However, the derivation of (3.31) made the generic assumption $f_{\lambda} \not \equiv 0$, This may be violated for $\lambda=0$. In this case the constant in (3.31) vanishes (i.e. $k_{0}=0$ ), and solutions related to PI (discussed in the next section) are obtained.

Almost identical arguments lead to the following equations for the taufunctions of the systems $\mathcal{H}_{(i)}$ and $\mathcal{H}_{(i i i)}$ :

$$
\begin{aligned}
z \tau_{\lambda}^{4}-6 \tau_{\lambda}^{2} D_{z}^{4} \tau_{\lambda} \cdot \tau_{\lambda}+9\left(D_{z}^{2} \tau_{\lambda} \cdot \tau_{\lambda}\right)^{2} & =\tilde{\tau}_{\lambda-\frac{3}{2}} \tilde{\tau}_{\lambda+\frac{3}{2}} \\
z \tilde{\tau}_{\lambda}^{2}-\frac{3}{4} D_{z}^{4} \tilde{\tau}_{\lambda} \cdot \tilde{\tau}_{\lambda} & =\tau_{\lambda-\frac{3}{2}} \tau_{\lambda+\frac{3}{2}} \tilde{\tau}_{\lambda} .
\end{aligned}
$$

We have used the same conventions and genericity assumptions as for $\mathcal{H}_{(i i)}$. Observe that these equations are no longer bilinear, but it is consistent to assign weight one to $\tau$ and weight two to $\tilde{\tau}$ in the two equations.

\section{Special Solutions}

\subsection{Rational Solutions}

The rational solutions of the Painlevé equations have been constructed in many different ways [2, 5, 23. The rational solutions of (2.18) follow a pattern very similar to those of PII. They may be generated by applying the Bäcklund transformation (3.13) repeatedly, starting with the solution $y_{\frac{1}{2}}=0$, to produce a sequence of solutions $y_{\lambda}$ for half-integer values of $\lambda$. It is obvious that the solutions thus generated must be rational, as the Bäcklund transformation only involves differentiations and algebraic operations. The 
Miura map (3.11) also yields the associated sequence of rational functions $w_{\lambda}$ (giving solutions to the system $\mathcal{H}_{(i i)}$ ). From an algorithmic point of view, it is much more convenient to calculate the rational solutions from their polynomial tau-functions $\tau_{\lambda}$, which can be obtained by iteratively solving (3.31). We present a few of these rational solutions, and their associated taufunctions, in table 1 . Note that it is sufficient to consider only the positive half-integer values of $\lambda$, because of the formula $y_{\lambda+1}=-y_{-\lambda}$.

\begin{tabular}{|c|c|c|c|c|c|}
\hline$\lambda$ & $1 / 2$ & $3 / 2$ & $5 / 2$ & $7 / 2$ & $9 / 2$ \\
\hline$y_{\lambda}$ & 0 & $-\frac{1}{z}$ & $-\frac{2}{z}$ & $\frac{-3\left(z^{5}+96\right)}{z\left(z^{5}-144\right)}$ & $\frac{-4\left(z^{15}-72 z^{10}+217728 z^{5}-1741824\right)}{z\left(z^{15}-1152\left(z^{10}-84 z^{5}+6048\right)\right)}$ \\
\hline$w_{\lambda}$ & 0 & $-\frac{2}{z^{2}}$ & $-\frac{6}{z^{2}}$ & $\frac{-12\left(z^{10}+432\left(z^{5}+8\right)\right)}{z^{2}\left(z^{5}-144\right)^{2}}$ & $\frac{-20 z^{3}\left(z^{15}+1008 z^{10}+944488 z^{5}-47542144\right)}{\left(z^{10}-1008\left(z^{5}+48\right)\right)^{2}}$ \\
\hline$\tau_{\lambda}$ & 1 & $z$ & $z^{3}$ & $z^{6}-144 z$ & $z^{10}-1008\left(z^{5}+48\right)$ \\
\hline
\end{tabular}

Table 1: Rational solutions for case (ii).

The solutions above can also be obtained as specializations of the wellknown sequence of rational solutions of the $\mathrm{mKdV} / \mathrm{KdV}$ hierarchy constructed in [4], and thus it is possible to express the polynomial tau-functions $\tau_{\lambda}$ as Wronskian determinants [20].

Rational solutions $y_{\lambda}$ of the equation (2.17), together with the Miurarelated $w_{\lambda}, W_{\lambda-\frac{3}{2}}$ (giving solutions to systems $\mathcal{H}_{(i),(i i i)}$ ) may be generated in a similar fashion, by applying the Bäcklund transformation (3.23). Some of these solutions are given in table 2. There is one sequence for the values $\lambda=3 j+\frac{1}{2}$ (for integer $j$ ), generated from the trivial solution $y_{\frac{1}{2}}=0$, and a second sequence for $\lambda=3 j-\frac{1}{2}$ (which is related to the first by (3.25)). Once again, it is easier to generate solutions using the equations (3.32,3.33) for the tau-functions. We present some of these in table 3 below (using two rows for the pair $\tilde{\tau}_{\lambda \pm \frac{3}{2}}$ related by $(3.32)$ ).

\subsection{Solutions Related to PI}

All three systems have special solutions related to (1.1), the first Painlevé transcendent (PI). If we consider the system $\mathcal{H}_{(i)}$ in the case $\lambda=0$, the same substitution that works in the ordinary (autonomous) system causes 


\begin{tabular}{|c|c|c|c|c|c|c|c|}
\hline$\lambda$ & $-7 / 2$ & $-5 / 2$ & $-1 / 2$ & $1 / 2$ & $5 / 2$ & $7 / 2$ & $11 / 2$ \\
\hline$y_{\lambda}$ & $\frac{4}{z}$ & $\frac{3\left(z^{5}-24\right)}{z\left(z^{5}+36\right)}$ & $\frac{1}{z}$ & 0 & $-\frac{2}{z}$ & $-\frac{3}{z}$ & $\frac{-5 z^{4}\left(z^{5}+216\right)}{\left(z^{5}+36\right)\left(z^{5}-144\right)}$ \\
\hline$w_{\lambda}$ & $-\frac{12}{z^{2}}$ & $-\frac{6}{z^{2}}$ & 0 & 0 & $-\frac{6}{z^{2}}$ & $-\frac{12}{z^{2}}$ & $\frac{-30 z^{3}\left(z^{5}+576\right)}{\left(z^{5}-144\right)^{2}}$ \\
\hline$W_{\lambda-\frac{3}{2}}$ & $-\frac{12}{z^{2}}$ & $\frac{-15 z^{3}\left(z^{5}-144\right)}{2\left(z^{5}+36\right)^{2}}$ & $-\frac{3}{2 z^{2}}$ & 0 & 0 & $-\frac{3}{2 z^{2}}$ & $-\frac{12}{z^{2}}$ \\
\hline
\end{tabular}

Table 2: Rational solutions for cases (i) and (iii).

\begin{tabular}{|c|c|c|c|c|}
\hline$\lambda$ & $1 / 2$ & $5 / 2$ & $7 / 2$ & $11 / 2$ \\
\hline$\tau_{\lambda}$ & 1 & $z$ & $z^{2}$ & $z^{5}-144$ \\
\hline$\tilde{\tau}_{\lambda-\frac{3}{2}}$ & 1 & 1 & $z$ & $z^{5}+36$ \\
\hline$\tilde{\tau}_{\lambda+\frac{3}{2}}$ & $z$ & $z^{5}+36$ & $z^{8}$ & $z^{16}-2^{7} .3^{2} z^{11}+2^{11} .3^{4} .11 z^{6}+2^{14} .3^{6} .11 z$ \\
\hline
\end{tabular}

Table 3: Polynomial tau-functions for cases (i) and (iii).

the equations of motion to separate. Putting

$$
Q_{ \pm}=q_{1} \pm q_{2}
$$

into Hamilton's equations for $h_{0}$, we find

$$
Q_{ \pm}^{\prime \prime}=-\frac{1}{2} Q_{ \pm}^{2}+\frac{z}{2}
$$

which (up to a scaling) is just two separate copies of PI. The corresponding solution to (2.17) is

$$
y_{0}=\left(\log \left[Q_{+}-Q_{-}\right]\right)^{\prime}
$$

where we assume that $Q_{+}$and $Q_{-}$are not equal. So by applying the Bäcklund transformation (3.23) we get the general solution to the system $\mathcal{H}_{(i)}$ for $\lambda=$ 
$3 j$, and to $\mathcal{H}_{(i i i)}$ for $\lambda=3\left(j+\frac{1}{2}\right)$, for all integers $j$. However, there is also the degenerate case $Q_{+}=Q_{-}$, for which

$$
f_{0} \equiv 0
$$

This implies that the inverse Miura map and the Bäcklund transformation both break down. However, it is still possible to obtain a sequence of special solutions for the same parameter values, and they are also related to PI. Similarly, starting from a degenerate solution $Y_{0}$, corresponding to

$$
F_{0} \equiv 0
$$

the Bäcklund transformation (3.23) gives a sequence of special solutions to the system $\mathcal{H}_{(i)}$ for $\lambda=3\left(j+\frac{1}{2}\right)$, and to $\mathcal{H}_{(i i i)}$ for $\lambda=3 j$. We explain these degenerate solutions in more detail for case (ii).

The degenerate case for all three systems is $\lambda=0, f_{0} \equiv 0$. So in case (ii) $w=w_{0}$ must satisfy

$$
w^{\prime \prime}+3 w^{2}-\frac{z}{2}=0,
$$

which is equivalent to PI (after rescaling $w$ and $z$ ). The inverse Miura map breaks down in this case. However, the ordinary Miura map means that $y=y_{0}$ satisfies the Riccati equation

$$
y^{\prime}+y^{2}+w_{0}=0
$$

This is linearized by setting $y=(\log [\tau])^{\prime}$, to yield

$$
\tau^{\prime \prime}+w_{0} \tau=0
$$

which (for each $w_{0}$ ) gives a one parameter family of solutions $y_{0}$ to (2.18). Because $f_{0} \equiv 0$, the Bäcklund transformation (3.13) breaks down for the solution $y_{0}$. However, we still have

$$
y_{\lambda+1}=-y_{-\lambda}
$$

and so we can safely apply the Bäcklund transformation to $y_{1}=-y_{0}$ to obtain a sequence of solutions for all integers $\lambda$, as well as corresponding solutions to the system $\mathcal{H}_{(i i)}$.

Remark. These degenerate solutions to (2.18) are the analogues of the Airy function solutions to PII (see 23] and some of the other references). The fact that solutions of PI give special solutions of (2.18) is a particular case of a result in [24]. 


\section{Conclusions}

We have found that the Hamiltonian formalism for stationary flows of certain integrable PDEs may be extended to the similarity solutions. For the particular examples we have considered, non-autonomous generalizations of integrable Hénon-Heiles systems are obtained. Applying the same technique to the travelling wave similarity solutions of (2.13) leads to Hénon-Heiles Hamiltonians with harmonic terms, while the scaling similarity solutions of Hirota-Satsuma and a related system correspond to non-autonomous versions of the integrable Hamiltonians with quartic potentials considered in [7, 8]; these examples appear in [20]. Our method should always work provided that the ODEs for the similarity solutions can be written in the form

$$
B_{w} f=0
$$

where $B_{w}$ is the scaled version of the Hamiltonian operator for the PDE, and $f$ is the gradient of the (scaled) Hamiltonian for the PDE plus some extra (possibly non-autonomous) terms (cf. section 2).

It seems to be a common phenomenon that equations of Painlevé type can be viewed as non-autonomous generalizations of autonomous, integrable Hamiltonian systems. For instance, the equations in [18] are non-autonomous versions of the integrable Neumann systems. This connection might be used to study the asymptotics of higher order Painlevé equations. Recently we have succeeded in extending our approach to the multi-phase similarity solutions of the kind considered by Newell [26], but this will be considered (together with the connection to isomonodromic deformations) in a forthcoming article 21].

\section{Acknowledgements}

This work was supported by an EPSRC studentship. It is a pleasure to thank my supervisor, Harry Braden, for all his help and encouragement. I would also like to thank Allan Fordy for many useful discussions.

\section{References}

[1] M.J. Ablowitz, A. Ramani and H. Segur, Nonlinear evolution equations and ordinary differential equations of Painlevé type, Lett. Nuovo Cim. 23, 333-338 (1978). 
[2] M.J. Ablowitz and A.S. Fokas, On a unified approach to transformations and elementary solutions of Painlevé equations, J. Math. Phys. 23 (1982).

[3] M.J. Ablowitz and P.A. Clarkson, Solitons, Nonlinear Evolution Equations and Inverse Scattering, Cambridge University Press (1991).

[4] M. Adler and J. Moser, On a Class of Polynomials connected with the Korteweg-deVries Equation, Commun. Math. Phys. 61, 1-30 (1978).

[5] H. Airault, Rational Solutions of Painlevé Equations, Stud. Appl. Math. 61, 31-53 (1979).

[6] O. Babelon and D. Bernard, From form factors to correlation functions. The Ising model, Phys. Lett. B288, 113-120 (1992).

[7] S. Baker, V.Z. Enolskii and A.P. Fordy, Integrable Quartic Potentials and Coupled KdV Equations, Phys. Lett. A201, 167-174 (1995).

[8] S. Baker, Squared Eigenfunction Representations of Integrable Hierarchies, PhD Thesis, University of Leeds (1995).

[9] R. Caboz, L. Gavrillov and V. Ravoson, Separability and Lax pairs for Hénon-Heiles system, J. Math. Phys. 34, 2385-2393 (1993).

[10] Y.F. Chang, M. Tabor and J. Weiss, Analytic structure of the HénonHeiles Hamiltonian in integrable and nonintegrable regimes, J. Math. Phys. 23, 531-538 (1982).

[11] P.A. Clarkson and M.D. Kruskal, New similarity solutions of the Boussinesq equation, J. Math. Phys. 30, 2201-2213 (1989).

[12] M.R. Douglas, Strings in Less Than One Dimension and the Generalized KdV Hierarchies, Phys. Lett. B238, 176-180 (1990).

[13] V.Z. Enolskii, D.V. Leykin and M. Salerno, A canonical transformation between integrable Hénon-Heiles systems, Phys. Rev. E49, 5897-5899 (1994).

[14] H. Flaschka and A.C. Newell, Monodromy- and Spectrum-Preserving Deformations I, Commun. Math. Phys. 76, 65-116 (1980).

[15] A.P. Fordy and J. Gibbons, Factorization of operators I, J. Math. Phys. 21, 2508-2510 (1980); Factorization of operators II, J. Math. Phys. 22, 1170-1175 (1981). 
[16] A.P. Fordy, The Hénon-Heiles system revisited, Physica 52D, 204-210 (1991).

[17] A.P. Fordy, Stationary flows: Hamiltonian structures and canonical transformations, Physica 87D, 20-31 (1995).

[18] J. Harnad, C.A. Tracy and H. Widom, Hamiltonian Structure of Equations Appearing in Random Matrices, NATO ASI Series B, Vol.314, 231245, Plenum Press (1993).

[19] A.N.W. Hone, Non-autonomous Hénon-Heiles Systems, Proceedings of the First Non-Orthodox School of Nonlinearity and Geometry, Warsaw (1995).

[20] A.N.W. Hone, Integrable Systems and their Finite-Dimensional Reductions, PhD Thesis, University of Edinburgh (1996).

[21] A.N.W. Hone, Hamiltonians for Multi-Phase Similarity Solutions, in preparation.

[22] A.R. Its, A.G. Izergin, V.E. Korepin and N.A. Slavnov, Differential Equations for Quantum Correlation Functions, Int. J. Mod. Phys. B, Vol. 4, No. 5, 1003-1037 (1990).

[23] K. Kajiwara and Y. Ohta, Determinant Structure of the Rational Solutions for the Painlevé II Equation, J. Math. Phys. 37, 4693-4704 (1996).

[24] N.A. Kudryashov, The first and second Painlevé equations of higher order and some relations between them, Phys. Lett. A224, 353-360 (1997).

[25] Y.I. Manin, Sixth Painlevé Equation, Universal Elliptic Curve and Mirror of $P^{2}$, alg-geom/9605010.

[26] A.C. Newell, Solitons in Mathematics and Physics,

[27] K. Okamoto, On the tau-function of the Painlevé equations, Physica 2D, 525-535 (1981); Studies on the Painlevé Equations III, Math. Ann. 275, 221-255 (1986).

[28] C. Rogers and R. Shadwick, Bäcklund transformations and their Applications, Academic Press (1982). 